\title{
Effect of monoamine oxidase B A644G variant on nicotine dependence and/or schizophrenia risk
}

\author{
Sacide Pehlivan¹, Pinar Cetinay Aydin², Mehmet Atilla Uysal³3, Hayriye Senturk Ciftci¹, Ulgen Sever¹, \\ Fatih Kasim YavuZ2, Nazan Aydin², Ayse Feyda Nursal ${ }^{4}$
}

1 Department of Medical Biology, Istanbul University, Istanbul Faculty of Medicine, Istanbul, Turkey.

2 Department of Psychiatry, Bakirkoy Research and Training Hospital for Psychiatry, Neurology and Neurosurgery, Istanbul, Turkey.

${ }^{3}$ Department of Chest Diseases, Yedikule Hospital For Chest Diseases and Thoracic Surgery Training and Research Hospital, Istanbul, Turkey.

${ }^{4}$ Department of Medical Genetics, Hitit University, Faculty of Medicine, Corum, Turkey.

Received: 10/24/2018 - Accepted: 11/28/2018

DOI: 10.1590/0101-60830000000186

\begin{abstract}
Objectives: Schizophrenia (Sch) is a severe and chronic mental illness. Smoking prevalence is higher in patients with Sch than general population. We aimed to investigate the effects of $M A O B$ gene $\mathrm{A} 644 \mathrm{G}$ variant on nicotine dependence (ND) and Sch+ND risk in Turkish population and to evaluate by bioinformatic analysis. Methods: Present study included 161 individuals with ND, 223 patients with Sch+ND, and 96 non-smoker controls. MAOB A644G variant was analyzed using PCR-RFLP method. As the MAOB gene is located on the X chromosome, each gender was analysed separately. Results: The total distributions of AA, AG and GG genotypes of $M A O B$ gene $\mathrm{A} 644 \mathrm{G}$ were $44.7 \%, 22.4 \%$ and $32.9 \%$ in the ND group, $45.3 \%, 25.1 \%$ and $29.6 \%$ in the Sch+ND group and, 44.8 , $22.9 \%$ and $32.3 \%$ in non-smoker controls. No significant differences were observed between groups for the MAOB A644G genotype and allele frequencies when female group compared to male group ( $p$ 0.05). Examination of disease associations of SNPs from each miRNA gene region in GWAS databases yielded results for aging, bipolar disorder, autoimmune, and neurological diseases. Discussion: Our results indicate that the MAOB gene A644G variant is not associated with ND and/or Sch susceptibility in the Turkish population.
\end{abstract}

Pehlivan S et al. / Arch Clin Psychiatry. 2019;46(1):21-4

Keywords: Schizophrenia, nicotine dependence, monoamine oxidase B, variant

\section{Introduction}

Schizophrenia ( $\mathrm{Sch}$ ) is a chronic and severe mental disorder with an elusive psychopathology. This disease can be extremely devastating and interferes with cognition, emotion, perception, and other behavioral aspects ${ }^{1}$. Inheritance has been found in up to $80 \%$ of the cases ${ }^{2}$, however, the exact etiology of this disorder is still unclear. Many researchers have proposed that dysregulated dopaminergic neurotransmission is involved in the pathogenesis ${ }^{3}$. Dopamine acts as a neurotransmitter by binding to its specific receptors on the postsynaptic membrane and autoreceptors on the presynaptic membrane.

Tobacco use is the only most preventable cause of death, disability and illness in the World. Nicotine, the major active component of cigarettes that plays a role in physical dependence, influences nicotine receptors in the central nervous system and results in the release of neurotransmitters (including dopamine). Nicotine activates dopaminergic neurons in the mesolimbic reward system and induces dopamine release ${ }^{4}$. The prevalence of smoking is much higher among people with psychotic disorders compared to the general population. Besides, among those suffering from $\mathrm{Sch}$, the nicotine dependence (ND) occurs more frequently than both the general population and those with other psychiatric diseases ${ }^{5}$.

Genes that play a role in neurotransmitter metabolism pathways have been widely examined in studies of psychiatric disorders. Monoamine oxidase (MAO; E.C. 1.4.3.4) is a flavinadenine-dinucleotide (FAD)-containing enzyme that plays a role in the metabolic breakdown of several biogenic amines 6 . It is found on the outer membrane of mitochondria in most cell types. Two isoenzymes (MAOA and $M A O B$ ) of $M A O$ are present. In humans, MAOA ideally oxidizes serotonin and noradrenaline, while $M A O B$ oxidizes dopamine ${ }^{7}$. MAOA and $M A O B$ genes are sequenced tail to tail on the Xp11.23 chromosomal region, and both genes have 15 homologous exons (1). A644G single nucleotide polymorphism (SNP) (rs1799836) in intron 13 of the $M A O B$ is known functional polymorphism8.
Therefore, we conducted a case-control study in a Turkish population, to assess the impact of the $M A O B$ A644G variant on risk ND and/or Sch+ND. As the $M A O B$ gene is located on the $\mathrm{X}$ chromosome, each gender was analyzed separately.

\section{Methods}

\section{Study population}

The study sample included 161 subjects with ND (87 male and 74 female; mean age: 45.93 years), 223 subjects with Sch+ND (105 male and 118 female mean age: 46.78 years), and 96 unrelated healthy controls (41 male and 55 female; mean age: 44.91 years). The ethnicity of all participants was Turkish. The patients with Sch were recruited from the Department of Psychiatry Clinic, Bakirkoy Mental Health Research and Training Hospital, Istanbul, Turkey and diagnosed according to the Diagnostic and Statistical Manual of Mental Disorders, 4th ed. (DSM-IV) ${ }^{9}$. ND group was selected from the Department of Chest Diseases, Yedikule Hospital for Chest Diseases and Thoracic Surgery Training and Research Hospital, Istanbul, Turkey. ND group consisted of active smokers. These subjects were defined as those who had previously smoked more than one cigarette per day but had quit smoking for more than one year. The degree of ND was evaluated by the scores on Heaviness of Smoking Index (HSI) and the Fagerström Test for Nicotine Dependence (FTND) ${ }^{10}$. Control group was recruited from "non-smokers" were defined as those who had smoked less than one cigarette per day for no more than 1 year during their lifetime. Before enrollment, signed informed consent was obtained from every participant or their guardians if the participant was a minor or could not provide consent. The study protocols were performed according to the principles of the Declaration of Helsinki. This study was approved by the Ethics Committees of the Istanbul University, Istanbul Medical Faculty. 


\section{Genotyping}

Blood samples were collected from all participants using EDTA tubes and DNA was extracted from whole blood using salting out method ${ }^{11}$. DNA samples were stored at $-20{ }^{\circ} \mathrm{C}$ until genotype analysis. The $M A O B$ A644G variant was analyzed by polymerase chain reaction (PCR) and restriction fragment length polymorphism (RFLP) assays ${ }^{12}$. The segment was replicated by using the following forward and reverse PCR primers: 5-GGAACCTCTTATACCACAGG-3 and 5-GACTGCCAGATTTCATCCTC-3. Then the DNA products was digested with the restriction enzyme Tsp 45I. MAOB allele 1 (containing A and therefore the Tsp 45I restriction site) was detected as two bands of 146 and $86 \mathrm{bp}$, whereas allele 2 (containing $\mathrm{G}$ and no Tsp $45 I$ restriction site) was detected as a single 232-bp band. Since the $M A O B$ gene is found on the $\mathrm{X}$ chromosome, i.e. males carry only one allele for $M A O-\mathrm{B}$.

\section{Bioinformatic analysis}

We examined the regulation of expression of $M A O B$ (microRNAs (miRNAs); co-regulated gene sets), and disease associations of $M A O B$ sequence variants. Gene co-expression networks can be used to associate genes of unknown function with biological processes, to prioritize candidate disease genes. We used the CO-Regulation Database (CORD) for co-expression analysis, GSEA/MSigDB for gene enrichment analysis, TargetScan for miRNAs targeting $M A O B$ and GRASP as GWAS Database for disease associations of $M A O B$ and miRNA region SNPs. Also, we used ${ }^{13}$ for enhancers effecting $M A O B$ expression and other genes.

\section{Statistical analysis}

The genotype distribution and allele frequency of the $M A O B$ A644G variant in the control and patient groups were compared using Chi-squared tests. The Hardy-Weinberg equilibrium (HWE) was calculated using the de Finetti program (Online HWE and
Association Testing-Institut für Humangenetik, Munich, Germany). Odds ratio (OR) and 95\% confidence intervals (CIs) were estimated using the binary logistic regression method. $p$ values less than 0.05 were considered statistically significant.

\section{Results}

In the present study, a total of 480 subjects, including 161 subjects with ND, 96 non-smoker control, and 223 patients with Sch+ND were genotyped for the $\mathrm{A} 644 \mathrm{G}$ variant of $M A O B$ gene. The genotype and allele frequencies of the $M A O B$ A644G variant in the studied groups was shown in table 1 . There were $74(45.9 \%)$ female and $87(54.1 \%)$ male in the ND group and $55(57.3 \%)$ female and 41 $(42.7 \%)$ male in the control group (non-smoker) and 118 (52.9\%) female and $105(47.1 \%)$ male in Sch+ND group. The prevalence of total genotypes of AA/A, AG, and G/GG profiles for MAOB A644G variant were $44.7 \%, 22.4 \%$ and $32.9 \%$ respectively in the ND group, $44.8 \%, 22.9 \%$, and $32.3 \%$ in non-smoker group, and $45.3 \%, 25.1 \%$ and $29.6 \%$ in the Sch+ND group.

The statistically significant relationship was not found between groups for the $M A O B$ A644G genotype and allele frequencies $(\mathrm{p}>0.05)$.

\section{Discussion}

Sch has been considered as a complex neurodevelopmental disorder. The brain's monoamine (dopamine, serotonin and norepinephrine) systems are involved in normal behavior and pathology within these circuits is suggested to underlie several neurological and psychiatric conditions. Dopamine neurons in the midbrain manifest various unique activity states that have implications for the function of the dopamine system. A dopamine hypothesis of Sch proposes a final common pathway of presynaptic striatal hyperdopaminergia resulted by an interaction between numerous environmental and genetic risk factors influencing brain function that lead to negative and cognitive symptoms ${ }^{3}$.

Table 1. Genotype and allele frequencies of MAOB A644G variant in ND, control and Sch+ND groups

\begin{tabular}{|c|c|c|c|c|c|c|c|}
\hline \multirow[t]{2}{*}{ MAOBA644G } & \multirow[t]{2}{*}{ Genotypes } & $\begin{array}{c}\text { ND } \\
\text { group }\end{array}$ & $\begin{array}{c}\text { Control } \\
\text { group }\end{array}$ & Sch+ND group & \multirow[t]{2}{*}{$\mathrm{OR}$} & \multirow[t]{2}{*}{$\% 95 \mathrm{Cl}^{*}$} & \multirow[t]{2}{*}{$p$} \\
\hline & & $\mathrm{n}(\%)$ & $\mathrm{n}(\%)$ & $\mathrm{n}(\%)$ & & & \\
\hline \multirow[t]{9}{*}{ Females } & $\mathrm{A} / \mathrm{A}$ & $24(32.4)$ & $17(30.9)$ & $41(34.7)$ & 1.030 & $0.751-1.413$ & $0.854 \mathrm{p}$ \\
\hline & & & & & 0.887 & $0.550-1.429$ & $0.753^{\mathrm{p} 2}$ \\
\hline & & & & & 0.937 & $0.638-1.377$ & $0.862^{\mathrm{p} 3}$ \\
\hline & $A / G$ & $36(48.7)$ & $22(40.0)$ & 56 (47.5) & 1.160 & $0.862-1.559$ & $0.425^{p}$ \\
\hline & & & & & 0.812 & $0.518-1.272$ & $0.451 \mathrm{p}^{2}$ \\
\hline & & & & & 1.031 & $0.720-1.472$ & $0.990^{\mathrm{p} 3}$ \\
\hline & $\mathrm{G} / \mathrm{G}$ & 14 (18.9) & $16(29.1)$ & $21(17.8)$ & 0.770 & $0.508-1.165$ & $0.208^{p}$ \\
\hline & & & & & 1.508 & $0.957-2.376$ & $0.136^{\mathrm{p} 2}$ \\
\hline & & & & & 1.047 & $0.666-1.645$ & $0.849^{3} 3$ \\
\hline \multirow[t]{3}{*}{ Males } & A & 48 (55.2) & 26 (63.4) & $60(57.1)$ & 0.898 & $0.709-1.137$ & $0.491 \mathrm{p}$ \\
\hline & G & 39 (44.8) & $15(36.6)$ & $45(42.9)$ & 1.209 & $0.702-2.082$ & $0.613^{\mathrm{p} 2}$ \\
\hline & & & & & 0.957 & $0.700-1.308$ & $0.898^{\mathrm{p}^{3}}$ \\
\hline \multirow[t]{9}{*}{ Total } & $A / A+A^{*}$ & $72(44.7)$ & 43 (44.8) & $101(45.3)$ & 0.858 & $0.718-1.025$ & $0.117 \mathrm{p}$ \\
\hline & & & & & 0.986 & $0.704-1.380$ & $0.994 \mathrm{p}^{2}$ \\
\hline & & & & & 0.939 & $0.743-1.189$ & $0.67 \mathrm{p}^{3}$ \\
\hline & $\mathrm{A} / \mathrm{G}$ & $36(22.4)$ & $22(22.9)$ & $56(25.1)$ & 0.998 & $0.786-1.241$ & $0.917 \mathrm{p}$ \\
\hline & & & & & 0.918 & $0.614-1.373$ & $0.782^{\mathrm{p} 2}$ \\
\hline & & & & & 0.914 & $0.685-1.218$ & $0.616^{\mathrm{p} 3}$ \\
\hline & $\mathrm{G} / \mathrm{G}+\mathrm{G}^{*}$ & $53(32.9)$ & 31 (32.3) & $66(29.6)$ & 1.011 & $0.728-1.235$ & $0.915 \mathrm{p}$ \\
\hline & & & & & 1.092 & $0.765-1.557$ & $0.728 \mathrm{p}^{2}$ \\
\hline & & & & & 1.093 & $0.853-1.400$ & $0.559 \mathrm{p}^{3}$ \\
\hline
\end{tabular}

ND: Nicotine dependence; Sch: schizophrenia; P1: ND versus control; P2: ND versus Sch+ND; P3: control versus Sch+ND. * The genotype distribution of MAOBA644G variant were compared as female, male and total (female+male) groups. 
Smoking is commonly encountered in Sch cases and it is one of the main causes of early death in this illness. Even though causes for such high frequency of cigarette smoking in patients with Sch remain unclear, dopamine seems to play a crucial role in this correlation. It has been shown that the addictive effects of smoking are not limited to the effects of nicotine, but may also be associated with the high dopamine levels ${ }^{14}$. Nicotine elevates dopamine levels in striatum by inducing its release via nicotinic receptors, and diminishing its breakdown by hindering monoamine oxidase type $\mathrm{A}$ and $\mathrm{B}^{15}$. Due to crucial role of dopamine in ND, variants in several dopamine related genes have been suggested and examined for their potent relations with smoking behaviour.

$M A O$ catalyses the oxidative deamination of numerous biogenic amines, and is involved in the regulation of neurotransmitters in the central nervous system, such as modulation of synaptic concentrations of serotonin, dopamine, norepinephrine and other catecholaminergic neurotransmitters. Enhancers affecting $M A O B$ expression and other genes: [EFHC2 (EF-hand domain containing 2) and MAOA are also targeted by the same enhancers affecting $M A O B$ expression]. An important paralog of this gene is MAOA. When 5-fold threshold was used, there were 192 concordantly co-expressed genes. (PHYH, ALDH6A1, RGN, FXYD1, MLXIPL, ISOC1, DDO, GSTM4, SLC6A12, AMACR, CRP, FLJ39378, FLJ21963). Using TargetScan we selected the miRNAs targeting $M A O B$ with a context score of less than -0.30, and determined their other target genes. Altogether, 26 miRNAs were identified as targeting $M A O B^{16}$.

Variations in $M A O$ enzyme expression and activity may contribute to genetic susceptibility for becoming a smoker, and could affect the ability of a smoker to quit. $M A O B$ A644G variant leads to a transitional conversion from adenine (A) to guanine $(G) 36$ bp upstream from the 5 -end of exon 14. It is proposed that, because of its position, this variant alters the stability and/or translation of $M A O B$ mRNA $^{17}$. Studies have been previously conducted to investigate the relation with smoking for polymorphisms of dopamine metabolic enzymes. Ito et al. found a decreased risk of being current smokers for individuals carrying the 4-repeat allele of the MAOA VNTR polymorphism in the promoter region for women, and the results were the opposite for men ${ }^{18}$. Costa-Mallen et al. suggested that $M A O B$ G644A was not associated with smoking status in either men or women, however, they reported that there was an interaction between $M A O B$ A644G and the D2 dopamine receptor (DRD2) gene TaqIB variants; they also proposed that subjects carrying $M A O B$ allele $\mathrm{A}$ and genotype $\mathrm{B} 12$ of $\mathrm{DRD} 2$ were 2.50 times more likely to be ever-smokers than the pool of men carrying all other genotype combinations ${ }^{19}$. Fowler et al. showed that both type A and type B $M A O$ are inhibited in the brains of smokers ${ }^{20}$. Also, it was found a significant association between the $M A O B$ intron 13 allele and different levels of $M A O B$ enzyme activity in human brain ${ }^{17}$. Tiili et al. reported that the $M A O B$ intron $3 \mathrm{AG}$ variant $\mathrm{G}$ allele was more prevalent in female smokers than non-smokers ${ }^{21}$. Also, it was reported that A allele in $M A O B$ G644A variant reduced the risk of heavy smoking in males ${ }^{22}$. However, Tochigi et al. obtained no evidence for an association between the MAO genes and smoking behavior, including epistatic or additive effects ${ }^{23}$.

There are several studies suggest that MAO genes may be implicated in the susceptibility to Sch. Investigated MAOA gene variants mostly haven't been linked with $\mathrm{Sc}^{24-25}$. For MAOB A644G variant and Sch, a sex specific association has been found in Spanish population, with a high prevalence of $G$ allele in women diagnosed with Sch compared to healthy women controls ${ }^{26}$. Although Peitl et al. reported that MAOA VNTR variant did not reveal statistically significant findings in overall samples, they found that MAOA VNTR variant allele 3 was statistically more frequent in male patients with $\mathrm{Sch}^{27}$. Bergen et al. reported that there was an association between a $M A O B$ microsatellite polymorphism and particular clinical features of patients with $\mathrm{Sch}^{28}$. In studies investigating Sch symptoms, Camarena was found that there were statistical differences between $M A O B$ A644G variant and affective flattening scores in patients with $\mathrm{Sch}^{29}$. But Zammit et al. supported that genotypes of MAOA and $M A O B$ genes are not risk factors for aggressive behavior in $\mathrm{Sch}^{30}$.

In present study, we investigated the possibility that $M A O B$ A644G variant may also be involved in predisposition to ND and ND+Sch. Since the $M A O B$ gene is found on the $\mathrm{X}$ chromosome, each gender was usually analyzed for both genders. To the best our knowledge, this is the first association study of $M A O B$ gene A644G and ND and/ or Sch risk in a Turkish population. The our results did not show the significant association between the genotype/allele frequencies of the $M A O B$ A644G variant and ND and/or Sch+ND risk.

The present study, however, bear some limitations. First, the findings may be limited to the Turkish population. Ethnic variations may also play a role in association studies. We could not determine $M A O B$ expression. Another limitation is that Sch subgroups are not specified. Furthermore, the present study has a relatively small population size and may not have the necessary strength. Therefore, larger sample sizes are needed to identify smaller effects, possibly of little clinical significance.

In conclusion, our preliminary findings could indicate the $M A O B$ A644G variant was not associated with ND and/or Sch+ND risk in a Turkish cohort. The complex nature of psychiatric illnesses mandates a multidisciplinary approach involving genetics, neuroscience, psychiatry, and molecular biology in order to achieve a final conclusion. As for now, we suggest that further large-scale studies should be conducted to clarify the role of this gene in ND and Sch pathogenesis to obtain more insight into this hypothesis.

\section{Acknowledgement}

This study was supported by Istanbul University BAP (BEK: 201725175 and TYL: 2414-50695) program.

\section{Informed consent}

Written informed consent was obtained from subjects and patients who participated in this study.

\section{References}

1. Wei YL, Li CX, Li SB, Liu Y, Hu L. Association study of monoamine oxidase A/B genes andschizophrenia in Han Chinese. Behav Brain Funct. 2011;7:42

2. Sullivan PF, Kendler KS, Neale MC. Schizophrenia as a complex trait: evidence from a meta-analysis of twin studies. Arch Gen Psychiatry. 2003;60(12):1187-92.

3. Howes OD, Kapur S. The dopamine hypothesis of schizophrenia: version III - The final common pathway. Schizophr Bull. 2009;35(3):549-62.

4. Jin Y, Chen D, Hu Y, Guo S, Sun H, Lu A, et al. Association between monoamine oxidase gene polymorphisms and smoking behaviour in Chinese males. Int J Neuropsychopharmacol. 2006;9(5):557-64.

5. de Leon J, Becoña E, Gurpegui M, Gonzalez-Pinto A, Diaz FJ. The association between high nicotine dependence and severe mental illness may be consistent across countries. J Clin Psychiatry. 2002;63(9):812-6.

6. Kang SG, Park YM, Choi JE, Lim SW, Lee HJ, Lee SH, et al. Association study between antipsychotic-induced restless legs syndrome and polymorphisms of monoamine oxidase genes in schizophrenia. Hum Psychopharmacol. 2010;25(5):397-3.

7. Shih JC, Chen K, Ridd MJ. Monoamine oxidase: from genes to behavior. Annu Rev Neurosci. 1999;22:197-17.

8. Garpenstrand H, Ekblom J, Forslund K, Rylander G, Oreland L. Platelet monoamine oxidase activity is related to MAOB intron 13 genotype. J Neural Transm (Vienna). 2000;107(5):523-30.

9. Cooper JE. On the publication of the diagnostic and statistical manual of mental disorders: fourth edition (DSM-IV). Br J Psychiatry. 1995;166:4-8.

10. Heatherton TF, Kozlowski LT, Frecker RC, Fagerström KO. The Fagerström test for nicotine dependence: a revision of the Fagerström tolerance questionnaire. Br J Addict. 1991;86(9):1119-27.

11. Miller SA, Dykes DD, Polesky HF. A simple salting out procedure for extracting DNA from human nucleated cells. Nucleic Acids Res. $1988 ; 16(3): 1215$ 
12. Torkaman-Boutorabi A, Ali Shahidi G, Choopani S, Reza Zarrindast M. Association of monoamine oxidase B and catechol-O-methyltransferase polymorphisms with sporadic Parkinson's disease in an Iranian population. Folia Neuropathol. 2012;50(4):382-9.

13. GeneCards. MAOB Gene (Protein Coding). Available from: http://www. genecards.org/cgi-bin/carddisp.pl?gene=MAOB\#genomic_location.

14. Fowler JS, Logan J, Wang GJ, Volkow ND. Monoamine oxidase and cigarette smoking. Neurotoxicology. 2003;24(1):75-82.

15. Sagud M, Mihaljević-Peles A, Mück-Seler D, Pivac N, Vuksan-Cusa B, Brataljenović T, et al. Smoking and schizophrenia. Psychiatr Danub. 2009;21(3):371-5.

16. Bioinformatıcs Tools. Available from: http://www.dorak.info/mtd/ bioinf.html.

17. Balciuniene J, Emilsson L, Oreland L, Pettersson U, Jazin E. Investigation of the functional effect of monoamine oxidase polymorphisms in human brain. Hum Genet. 2002;110(1):1-7.

18. Ito H, Hamajima N, Matsuo K, Okuma K, Sato S, Ueda R, et al. Monoamine oxidase polymorphisms and smoking behaviour in Japanese. Pharmacogenetics. 2003;13(2):73-9.

19. Costa-Mallen P, Costa LG, Checkoway H. Genotype combinations for monoamine oxidase-B intron 13 polymorphism and dopamine D2 receptor TaqIB polymorphism are associated with ever-smoking status among men. Neurosci Lett. 2005;385(2):158-62.

20. Fowler JS, Volkow ND, Wang GJ, Pappas N, Logan J, MacGregor R, et al. Inhibition of monoamine oxidase B in the brain of smokers. Nature. 1996;379(6567):733-36.

21. Tiili EM, Mitiushkina NV, Sukhovskaya OA, Imyanitov EN, Hirvonen AP. The genotypes and methylation of MAO genes as factors behind smoking behavior. Pharmacogenet Genomics. 2017;27(11):394-401.
22. Yang X, Chen H, Li S, Wang Q, Pan L, Jia C. Association between monoamine oxidase gene polymorphisms and smoking behavior: a meta-analysis. Drug Alcohol Depend. 2015;153:350-4.

23. Tochigi M, Suzuki K, Kato C, Otowa T, Hibino H, Umekage T, et al Association study of monoamine oxidase and catechol-O-methyltransferase genes with smoking behavior. Pharmacogenet Genomics. 2007;17(10):867-72.

24. Jönsson EG, Norton N, Forslund K, Mattila-Evenden M, Rylander G, Asberg M, et al. Association between a promoter variant in the monoamine oxidase A gene and schizophrenia. Schizophr Res. 2003;61(1):31-7.

25. Shi YZ, Wang CH, Lv LX, Wang YH, Zhang HX, Lou BY. [Association study of the polymorphisms of monoamine oxidase A genes with schizophrenia]. Zhonghua Yi Xue Yi Chuan Xue Za Zhi. 2007;24(4):457-9.

26. Gassó P, Bernardo M, Mas S, Crescenti A, Garcia C, Parellada E, et al. Association of $\mathrm{A} / \mathrm{G}$ polymorphism in intron 13 of the monoamine oxidase $B$ gene with schizophrenia in a Spanish population. Neuropsychobiology. 2008;58(2):65-70.

27. Peitl V, Štefanović M, Karlović D. Depressive symptoms in schizophrenia and dopamine and serotonin gene polymorphisms. Prog Neuropsychopharmacol Biol Psychiatry. 2017;77:209-15.

28. Bergen SE, Fanous AH, Walsh D, O'Neill FA, Kendler KS. Polymorphisms in SLC6A4, PAH, GABRB3, and MAOB and modification of psychotic disorder features. Schizophr Res. 2009;109(1-3):94-7.

29. Camarena B, Fresán A, Aguilar A, Escamilla R, Saracco R, Palacios J, et al. Monoamine Oxidase A and B Gene Polymorphisms and Negative and Positive Symptoms in Schizophrenia. ISRN Psychiatry. 2012;2012:852949.

30. Zammit S, Jones G, Jones SJ, Norton N, Sanders RD, Milham C, et al. Polymorphisms in the MAOA, MAOB, and COMT genes and aggressive behavior in schizophrenia. Am J Med Genet B Neuropsychiatr Gene. 2004;128B(1):19-20. 